www.jmscr.igmpublication.org

Impact Factor 5.84

Index Copernicus Value: 83.27

ISSN (e)-2347-176x ISSN (p) 2455-0450

crossref DOI:_https://dx.doi.org/10.18535/jmscr/v5i5.39

\title{
Clinical Profile of Pediatric Tuberculosis and MGIT sensitivity: A Prospective Study from a Tertiary care Institute
}

\author{
Authors \\ Ashok Garg ${ }^{1}$, Rakesh Sharma ${ }^{2}$, Jyotsna Sharma ${ }^{3}$, Pancham Kumar ${ }^{4}$, \\ Sandhya Chauhan ${ }^{5}$, Deepak Sharma ${ }^{6}$ \\ ${ }^{1}$ Junior Resident, ${ }^{2}$ Professor, ${ }^{3}$ Senior Resident, ${ }^{4}$ Assistant Professor, \\ ${ }^{5}$ Junior Resident, ${ }^{6}$ Medical student \\ 1,2,3,4 Department of Paediatrics, IGMC, Shimla-171001, H.P, India \\ ${ }^{5}$ Deparment of Dermatology, Venereology and Leprosy, IGMC, Shimla, H.P. India \\ ${ }^{6}$ Medical student, MMU, Solan, H.P. India \\ Corresponding Author \\ Ashok Garg \\ Email: ashokgarg44@gmail.com
}

\section{ABSTRACT}

Introduction: Tuberculosis (TB) is a major global health problem. Childhood tuberculosis (TB) is common in our community but it is relatively neglected, due to greater challenges in diagnosis. Clinical manifestations of childhood TB differ from adults. The diagnosis in most cases is based on clinical evidence but chest X-ray, Mantoux test and sputum/gastric sample microscopy are important supporting investigations.

Objective

1) To study the clinical profile of TB.

2) To determine incidence of extra-pulmonary $T B$ in children.

3) To know MGIT sensitivity in diagnosis of Tuberculosis.

Methods: This prospective study was conducted among admitted and OPD patients in the department of Pediatrics over a period of 1 year. Clinically suspected cases of TB in the age group 0-14 years who met the diagnostic criteria made the study group. Refusal of consent by parent and children already on TB treatment were excluded from this study. Investigations like chest X-ray, Mantoux test, sputum/gastric aspirate microscopy and MGIT were done to confirm the clinical diagnosis.

Results: The incidence of tuberculosis was found 36/10000. Pulmonary tuberculosis was more common (53.9\%) than extra pulmonary (35.3\%) tuberculosis. BCG scar was present in $87.42 \%$. History of contact was present in $41.2 \%$ and Mantoux test was positive in $51.98 \%$. MGIT sensitivity was $30 \%$ in our study. The most common symptoms were fever (79.4\%), anorexia (61\%) and cough (59\%).

Conclusion: This study supports that detailed history, clinical evaluation and active investigative workup has a major role in diagnosing childhood tuberculosis.

Keywords: Tuberculosis, Children, Mantoux, BCG, MGIT. 


\section{Introduction}

Tuberculosis (TB) is a major global health problem. It causes ill health among millions of people each year and ranks alongside the human immunodeficiency (HIV) as a leading cause of death worldwide. ${ }^{[1]}$ Childhood tuberculosis remains an important cause of morbidity and mortality in developing countries. ${ }^{[2]}$ Although data on childhood TB is scarce, tuberculin surveys conducted in children suggest a high rate of infection in the community with annual risk of TB infection is about $1.5 \% .^{[3]}$ The high prevalence and incidence of TB in developing countries is probably due to a high prevalence of TB in adults acting as contacts, poor socioeconomic condition, malnutrition, overcrowding and HIV coinfections. ${ }^{[4,5,6]}$

Since the declaration by WHO of a 'Global TB emergency' in 1993, a wealth of publications have addressed important aspects of the burden, management and control of tuberculosis. In general, however, the emphasis has been on adult disease. By contrast, the pediatric TB has been relatively neglected, mainly by the challenges in the diagnosis and the lower priority by TB control programs. While the burden of TB in India is not known, regional data from WHO indicate that the sputum smear positive TB in children $<14$ years old accounts for $0.6 \%$ to $3.6 \%$ of all reported cases. However, because the majority of children are sputum smear negative, this data underestimate the true burden of childhood TB. It is estimated that childhood TB constitutes $10-20 \%$ of all TB in high burden countries and accounts for $8-10 \%$ TB related deaths. ${ }^{[7]}$ Literature on tuberculosis in children is scarce in the developing countries including India. Further, no such study has been done earlier in Himachal Pradesh, so our aim was to determine the clinical profile of TB in children between 0-14 years of age.

\section{Aims and Objectives}

1. To study the clinical profile of $\mathrm{TB}$ in children 0-14 years age group.
2. To determines the incidence of extra pulmonary TB in children.

3. To study MGIT sensitivity in diagnosis of tuberculosis.

\section{Material and Methods}

It is a prospective observational type of study carried out over a period of one year in both outpatient and inpatient population in department of pediatrics. All patients in 0-14 year age group with features of tuberculosis on history and physical examination, patients with strong history of contact with tuberculosis were included in this study. Informed consent was taken from all patients. Cases were assessed clinically by taking relevant history and doing physical examination. Specific investigations were undertaken to establish the diagnosis of tuberculosis. Categorization of patients was done in two groups, pulmonary TB and extra pulmonary TB based on criteria described below.

For pulmonary tuberculosis:

1. Sputum or gastric aspirate positive for AFB my Zeihl-Neelsen method

Or

2. Two or more of the following:

- History of contact with a TB patient

- Cough lasting longer than 2 weeks

- Reactive tuberculin skin test

- Radiographic findings compatible with TB

- Response to anti-TB therapy (increased bodyweight by $10 \%$ after 2 months, decrease in symptoms)

For extrapulmonary TB:

- Fine needle aspiration cytology (FNAC)/ Histopathological evidence of $\mathrm{TB}$ in biopsy from lymph nodes, synovium or as relevant, or

- Bacteriological evidence of TB on microscopy or culture of serous fluids including pleural, pericardial, ascitic, synovial fluid and CSF, or

O History suggestive of TB including positive contact history, past history of TB or of anti-tubercular treatment, with 
clinical signs suggestive of $\mathrm{TB}$ on examination with strong corroborative investigative evidence in the form of 1 or more of the following

- A reactive tuberculin test

- Radiographic findings compatible with TB

- Lymphocytosis in cytology of serous fluids including pleural, pericardial, ascitic, synovial fluid and CSF.

- Adenosine deaminase levels suggestive of TB in serous fluids including pleural, pericardial, ascitic, synovial fluid and CSF.

- CT/ MRI suggestive of tuberculoma or tuberculous meningitis.

- Ultrasound abdomen suggestive of abdominal TB, Or,

- Response to anti-TB therapy (increased body weight by $10 \%$ after 2 months, decrease in symptoms).

After the clinical diagnosis, the disease was classified as per the system involved. Investigations like tuberculin skin test, sputum/gastric aspirate for AFB and MGIT-960 were done for confirmation of clinical diagnosis. Radiological investigations like chest X-ray, USG abdomen (for abdominal TB) and CT/MRI were performed in relevant cases. Specific condition like lymphadenitis required FNAC, for pleural, pericardial and peritoneal effusions, fluid aspiration studies were done and samples were sent for bacteriological confirmation and biochemical profile. In meningitis lumbar puncture was done and CSF was sent for bacteriological confirmation and biochemical profile. CT/MRI was done in cases of suspected tuberculoma.

\section{Observations}

During one-year study period, 28200 children visited our hospital including both outdoor and indoor patients, of theses 102 were diagnosed as TB. Incidence of TB was calculated as 36 per 10000 children.

Out of 102 cases, $50.9 \%$ were males and $49.1 \%$ were females. Male to female ratio was 1.04.

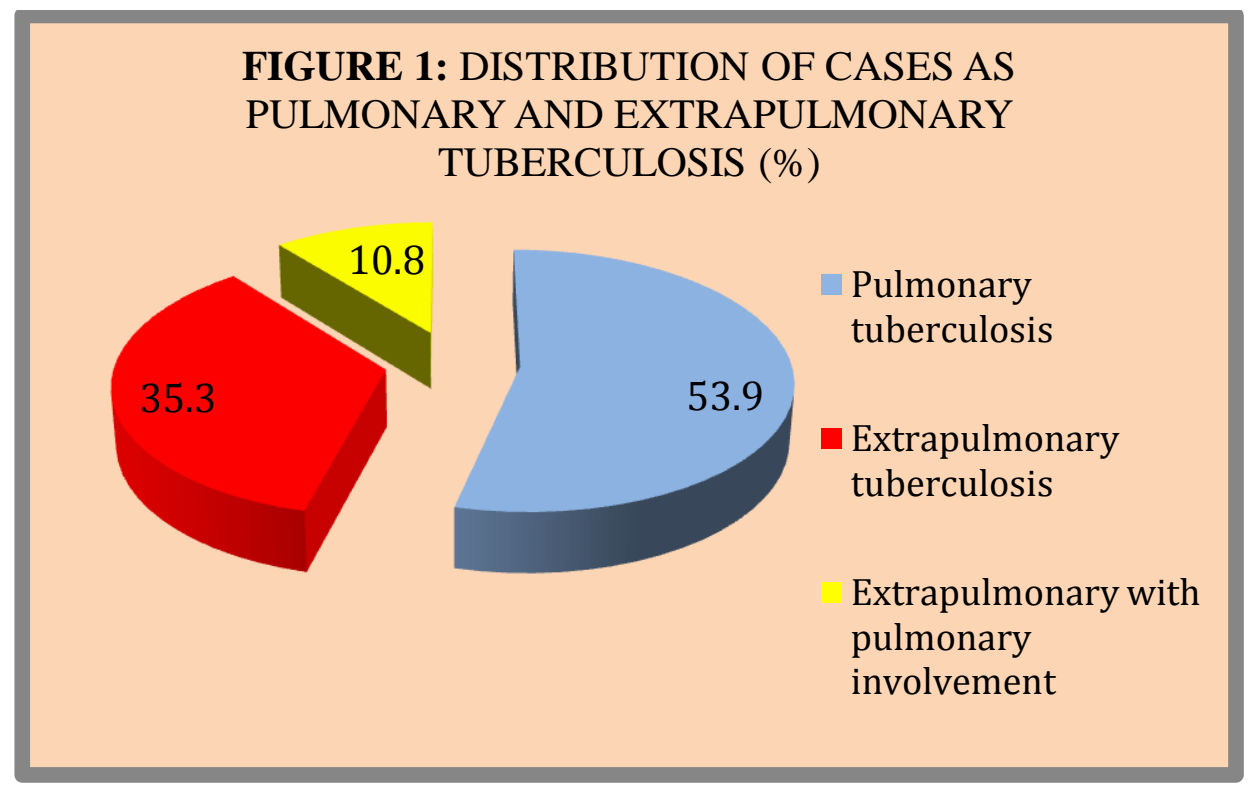

Table 1: Demographical profile of children

\begin{tabular}{|l|c|c|c|}
\hline Age group & Male (\%age) & Female(\%age) & Total(\%age) \\
\hline $0-5$ & $13(54.2)$ & $11(45.8)$ & $24(100)$ \\
\hline $6-10$ & $10(55.6)$ & $8(44.4)$ & $18(100)$ \\
\hline $11-14$ & $29(48.3)$ & $31(51.7)$ & $60(100)$ \\
\hline total & $52(50.9)$ & $50(49.1)$ & $102(100)$ \\
\hline
\end{tabular}




\section{JMSCR Vol||05||Issue||05||Page 21447-21453||May}

Mean age of TB affected children was 10.3 years. Most cases belonged to socioeconomic class IV and V $(50 \%)$ followed by middle class $(44.1 \%)$ and least number of cases were of upper class (3.9\%).

Positive family history of contact was found in 42 patients $(41.2 \%)$, whereas no history of contact was found in 60 patients $(58.8 \%)$. Most of cases $18(14.2 \%)$ with history of contact were found in age group $0-5$ years followed by 15 (35.7\%) cases in age group 11-14 years. Least number of cases 9 $(21.4 \%)$ were found in age group 6-10 years.

Table 2: Presenting symptoms in TB patients

\begin{tabular}{|l|c|c|c|}
\hline Serial number & Symptoms & No of cases & Percentage \\
\hline 1 & Fever & 81 & 79.4 \\
\hline 2 & Anorexia & 63 & 61.7 \\
\hline 3 & Cough & 59 & 57.8 \\
\hline 4 & Weight loss & 41 & 40 \\
\hline 5 & Headache & 17 & 16.6 \\
\hline 6 & Vomiting & 13 & 12.7 \\
\hline 7 & Chest pain & 11 & 10.7 \\
\hline 8 & Abdominal pain & 10 & 9.8 \\
\hline 9 & Dyspnea & 9 & 8.8 \\
\hline 10 & Seizure & 9 & 8.8 \\
\hline 11 & Neurological deficit & 2 & 1.9 \\
\hline
\end{tabular}

Table 3: Clinical signs in TB patients

\begin{tabular}{|l|c|c|c|}
\hline S.no & Clinical signs & Number & $\%$ \\
\hline 1. & Pallor & 31 & 30.4 \\
\hline 2. & Lymphadenopathy & 23 & 22.5 \\
\hline 3. & Pleural effusion & 15 & 14.7 \\
\hline 4. & Meningeal signs & 9 & 8.8 \\
\hline 5. & Signs of raised ICT & 7 & 6.9 \\
\hline 6. & Hepatosplenomegaly & 5 & 4.9 \\
\hline 7. & Doughy abdomen & 5 & 4.9 \\
\hline 8. & Neurological deficit & 2 & 1.9 \\
\hline 9 & Respiratory distress & 2 & 1.9 \\
\hline 10. & Ascites & 2 & 1.9 \\
\hline 11. & Pericarditis & 1 & 0.9 \\
\hline 12. & Arthritis & 0 & 0 \\
\hline
\end{tabular}

In relation to sex wise distribution, pulmonary tuberculosis was found in $29.4 \%$ males in comparison to $24.5 \%$ females. Extrapulmonary tuberculosis affected $14.7 \%$ males and $20.6 \%$ in females.

Amongst extra pulmonary TB, abdominal TB was most common type accounting for $11.8 \%$ cases, whereas pericardial TB was least common variant in $0.9 \%$ cases.

Amongst investigations, AFB positivity in sputum/gastric aspirate was detected in 31(30.4\%) cases, while it was negative in 71 cases $(69.6 \%)$. Maximum AFB positive cases were found in age group 11-14 years (25.5\%) followed by $25.5 \%$ cases in 6-10 years age group.
Chest x-rays of 102 cases were done, out of them $69.6 \%$ showed lesion on $X$ ray film. Primary complex was most common finding observed in $35.4 \%$ cases, pleural effusion was seen in $14.7 \%$, infiltrates in $9.8 \%$, miliary and cavitary pattern were appreciated in $3.9 \%$ cases for each.

BCG vaccination was given in $87.42 \%$ cases ( 88 out of 102 cases) and $13.56 \%$ (14 out of 88 ) of those vaccinated did not have a BCG scar on examination.

Mantoux test was positive in $53(51.98 \%)$ cases and $48.02 \%$ were negative. Maximum positive cases were in pulmonary TB $(30.4 \%)$, followed by pleural effusion (10.8\%). Abdominal TB, CNS TB, Disseminated TB each accounted for $2.94 \%$. 
MGIT was done in 30 patients, 9 patients were MGIT positive while 21 being MGIT negative. Sensitivity of MGIT was found to be $30 \%$. Maximum positive cases of MGIT were found in pulmonary TB followed by Disseminated TB.

\section{Discussion}

The incidence of tuberculosis was 36 cases per 10000 patients. Incidence of pulmonary and extra pulmonary TB was 19 and 13 per 10000 patients. The incidence of TB in India in year 2014 was 12.6/10000. As diagnosis of childhood TB is very challenging in community, so most cases of suspected TB are referred to tertiary care centre. This can be the reason to explain the higher incidence of tuberculosis in our study than the actual incidence in India. Sex wise distribution of cases showed a slight male preponderance (50.9\%), which is similar to study by Franco Et al in Brazil which showed a male preponderance of $51.6 \% .{ }^{[8]}$ Sivanandan $\mathrm{S}{ }^{[9]}$ and Xi-Rong Wu et al [10] also observed a male preponderance of $63 \%$ and $58 \%$ respectively.

Majority of cases in our study belonged to age group 11-14 years of age which is in accordance to study by Kakarani with $54 \%$ cases in age group 6-12 years. ${ }^{[11]}$ Analyzing and comparing the above studies with the present study, it may be concluded than in early adolescence there is a slight female preponderance. The increased risk may be due to combination of increased biological susceptibility, immunological response following puberty or difference in socialization patterns of male compared to female adolescents. Most of cases belonged to lower (50\%) and middle (44.1\%) class. A study on children from south India showed similar results with $55.8 \%$ belonging to lower class, $38.9 \%$ middle class, and $5.3 \%$ to high socioeconomic group. ${ }^{[12]}$

The frequency of clinical symptoms was observed as fever in $79.4 \%$, anorexia $63 \%$, cough $57.8 \%$ and weight loss in $40 \%$. Similar observations were reported in a study from north India by Garg P with fever and anorexia being commonest symptoms followed by weight loss and cough. ${ }^{[13]}$
In a study by Franco et al ${ }^{[8]}$ fever was documented in $73.1 \%$ cases and weight loss in $53.1 \%$. Positive family history of contact was found in 42 patients $(41.2 \%)$, most number of cases $18(42.9 \%)$ were in age group 0-5 years. This was similar to various Indian studies done in children under 15 years of age showing positive history of contact in $33-52 \%$ cases. ${ }^{[12,13,14]}$ Study by Franco et al ${ }^{[8]}$ reported a recent contact in age group 1-5 years.

In our study pulmonary TB constituted the major group (53.9\%), followed by extra pulmonary TB $(35.3 \%)$. Our results are consistent with the various studies described in literature ${ }^{[8,9,11,13,15]}$

In our study CNS tuberculosis accounted for $11.8 \%$ cases, this was comparable with study of Pontual et al ${ }^{[16]}$ where $16.67 \%$ cases were had isolated CNS tuberculosis. Lymphadenopathy was seen in $23.5 \%$ and isolated lymphadenopathy was seen in $1.96 \%$ cases. This was comparable to study done by Garg P, which showed $16.7 \%$ cases of tubercular lymphadenopathy ${ }^{13}$. Franco et al ${ }^{[8]}$ showed $21.5 \%$ cases of tubercular lymphadenopathy.

Primary complex was most common finding on $\mathrm{X}$ ray accounting for $36(50.7 \%)$ cases and $15(21.1 \%)$ cases showed pleural effusion. Similar to study by Franco et al ${ }^{[8]}$ observed primary complex in $44.3 \%$ cases.

History of BCG vaccination was positive in $87.42 \%$ cases and $13.72 \%$ of those vaccinated did not have a BCG mark on examination. Higher vaccination coverage in our study in comparison to others indicates effective implementation of National Immunization Programme. 
Table 4: Studies showing BCG coverage

\begin{tabular}{|l|c|c|c|c|}
\hline Serial no & Study & Year & No of cases & \%vaccinated \\
\hline 1. & Somu et al $^{15}$ & $1977-92$ & 11568 & 37.82 \\
\hline 2. & Garg p $^{13}$ & 1991 & 170 & 55.2 \\
\hline 3. & Sivanandan et al $^{9}$ & 2008 & 541 & 76 \\
\hline 4. & Present study & $2014-2015$ & 102 & 87.42 \\
\hline
\end{tabular}

Out of 102 cases, Mantoux test was positive in $53(51.98 \%)$ cases. Maximum positivity was seen in pulmonary $\mathrm{TB}(30.4 \%)$ and least was in disseminated TB, which accounted for $2.94 \%$. Sivanandan et al ${ }^{[9]}$ reported Mantoux positivity in $66 \%$ cases which is similar as to our study.

In present study, sputum/gastric aspirate microscopy was positive for AFB in 31(30.4\%) cases. This was in accordance to study done by Franco et al, ${ }^{[8]}$ which showed sputum positivity in $32.8 \%$ cases, and frequency of sputum positivity was higher in individual aged 11-15 years. Maximum sputum positivity i.e $25.5 \%$ was seen in age group 11-14 years followed by $4.9 \%$ in $6-10$ years. This finding emphasizes that as age increases percentage of sputum positivity increases and hence bacteriological sputum examination is a useful method above 10 years.

The reported sensitivity of MGIT is upto $90 \%$ and most of these studies were done in adults. The sensitivity of MGIT in our study was $30 \%$, which was in accordance to various studies showing sensitivity of $48.5 \%{ }^{[17]}, 21.9 \%{ }^{[18]}$ and $15 \% .^{[19]}$ The reason low sensitivity of MGIT in children was attributed to following factors- as children less than 8 years could not expectorate so we used gastric lavage, pleural fluid, CSF and these samples have low bacterial counts. Other factors like delay in transportation, storage and neutralization of sample would have inactivated tubercle bacilli. (as MGIT detects live bacteria)

\section{Conclusion}

Tuberculosis is a major global health problem. Most of work is done in the field of adult TB. Pediatric TB has been relatively neglected mainly due to greater challenges in diagnosis and lower priority tradionally afforded to children by TB control programmes. Detailed clinical evaluation, refinement of existing investigations and development/testing of new methods like MGIT are promptly required to improve diagnosis and treatment of TB in children.

\section{References}

1. Kochi A. The global tuberculosis situation and the new control strategy of the World Health Organization. Tuberc 1991;72:1-6.

2. Raviglione MC, Snider DE, Kochi A. Global epidemiology of tuberculosis. JAMA 1995;273:220-226.

3. Chadha VK, Agaewal SP, Kumar P, Chauhan LS, Kollapan C, Jaganath PS, et al. Average annual risk of tuberculosis infection in India. Ind $\mathbf{J}$ tuberc lung dis 2005;9:116-118.

4. Salazar GE, Schmitz TL, Cama R, Sheen P, Franchi LM, Centeno G, et al. Pulmonary Tuberculosis in Children in a Developing Country. Pediatric 2001;108:448453.

5. Stein brook R. HIV in India - a complex epidemic. N Eng J Med 2007; 356:10891093.

6. Kabra SK, Lodha R, Seth V. Category based treatment of tuberculosis in children. Indian Pediatric 2004;41:927-937.

7. Jain SK, Ordonez A, Kinikar A, Gupta A, Thakar M, Mave V, et al. Pulmonary Tuberculosis in Children in India: A Prospective study. Biomed research international volume 2013, Article ID 783698, 7 pages.

8. Franco R, Santana M. Clinical and Radiological Analysis of Children and Adolescents with Tuberculosis in Bahia, Brazil. The Brazilian journal of infectious diseases 2003;7;73-81. 
9. Sivanandan S, Walia M, Lodha R, Kabra SK. Factors associated with treatment failure in childhood tuberculosis. Indian Pediatr 2008;45:769-771.

10. Xi-Rong Wu, Qing-Qin Yin, An-Xia Jiao, Bao-Ping $\mathrm{Xu}$, Lin Sun, Wei-Wei Jiao et al. Pediatric Tuberculosis at Beijing Children's Hospital. Pediatrics;2012:130.

11. Kakarani VA, Pratinidhi AK. A study of childhood tuberculosis. Indian $\mathrm{J}$ Tub 1992;39:177-180.

12. Bai SS, Devi RL. Clinical spectrum of tuberculosis in BCG vaccinated children. Indian Pediatric 2002;39:458-62.

13. Garg P. Childhood tuberculosis in a community hospital from a region of high environmental exposure in north India. Journal of clinical and diagnostic research 2008:2:634-638.

14. Vijaysekaran D. Mantoux and contact positivity in tuberculosis. Indian J Pediatr 2006;73:989-993.

15. Somu N, Vijayasekaran D, Ravikumar T, Balachandran A, Subramanyam L. (1994) Tuberculous disease in a pediatric referral centre: 16 years' experience. Indian Pediatr 1994;31:1245-1249.

16. de Pontual L, Balu L, Ovetchkine P, Maury-Tisseron B, Lachassinne E, Cruaud P, et al. Tuberculosis in adolescents: a French retrospective study of 52 cases. Pediatr Infect Dis J 2006;25:930-932.

17. Gomez pastrana D. Diagnosis of pulmonary tuberculosis in children. Journal of infectious disease and therapeutics. 2013;1:17-24.

18. Rishi S, Sinha P, Malhotra B, Pal N. A comparative study for the detection of Mycobacteria by BACTAC MGIT 960, Lowenstein Jensen Media and Direct AFB smear examination. Indian Journal Med Microbiology 2007;25:383-386.

19. Siddiqui1 MAM, Anuradha PR, Nagamani $\mathrm{K}$, Vishnu PH. Comparison of conven- tional diagnostic modalities, BACTEC culture with polymerase chain reaction for diagnosis of extra-pulmonary tuberculosis. J Med Allied Sci 2013;3:53-58. 